\title{
ADVANCES IN TOTAL SYNTHESIS OF ALTERNARIC ACID: AN ANTI-FUNGAL AND PHYTOTOXIC NATURAL PRODUCT
}

\author{
SAJJAD AHMAD ${ }^{\prime}$, MUHAMMAD YOUSAF², AMEER FAWAD ZAHOOR ${ }^{2}$, , MATLOOB AHMAD ${ }^{2}$, ZULFIQAR ALI \\ KHAN² AND MUHAMMAD ARSLAN RASHID
}

\author{
${ }^{1}$ Department of Chemistry, University of Engineering and Technology Lahore, Faisalabad Campus, Faisalabad-38000, Pakistan \\ ${ }^{2}$ Institute of Chemistry, Government College University, Faisalabad-38000, Pakistan
}

\begin{abstract}
:
Alternaric acid is characterized as 1,4-diene, possessing (E)-1,2-disubstituted alkene known for nanomolar phytotoxic and antifungal activities. This article highlights the major synthetic approaches attempted towards the total synthesis of alternaric acid reported by three groups (Ichihara $\&$ coworkers, Trost $\&$ coworkers and Johnson and Slade) which involved novel pathways for the rapid construction of this functionalized natural molecule.
\end{abstract}

Keywords: Alternaric acid, natural products, anti-fungal, phyto-toxic, Alternaria solani

\subsection{INTRODUCTION:}

Alternaric Acid 1 was initially isolated in 1949 from stains of Alternaria solani by Brian and coworkers ${ }^{1,2}$ Alternaria solani is known as a fungus of early blight disease on tomato and potato plants and generates several secondary metabolites ${ }^{3}$. Alternaric acid $\left(\mathrm{C}_{21} \mathrm{H}_{30} \mathrm{O}_{8}, \mathrm{~m} . \mathrm{p} .138{ }^{\circ} \mathrm{C}\right)$ is a colorless, crystalline, optically inactive and unsaturated dibasic acid. It can be easily hydrolyzed by boiling dilute mineral acids or in sodium hydroxide however it remains unchanged in hot water ${ }^{4}$.

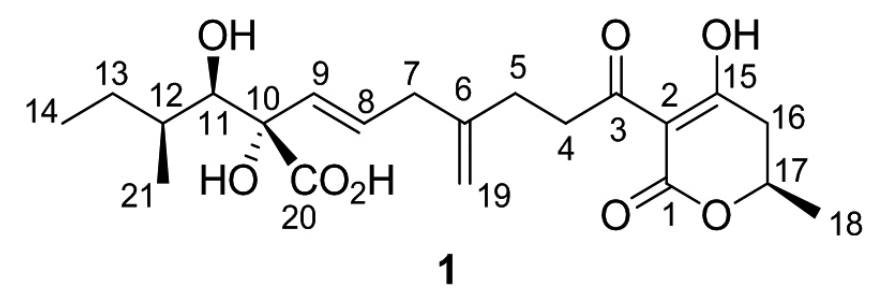

Figure 1: Alternaric Acid

Alternaric Acid $\mathbf{1}$ is considered to possess antifungal and phototoxic activities and accountable for plant diseases caused by . solani $^{3}$. It is also involved in inhibiting the event of hypersensitive death of potato cells tainted by an incompatible race of Phytophthora infestans ${ }^{5}$. It is known for suppression of germination of fungi like Absidia glauca, Myrothecium verrucaria spores at $1 \mu \mathrm{g} . / \mathrm{ml}$ or less of alternaric acid ${ }^{1}$. In 1960, chemical structure of alternaric acid was explored via classical chemical approaches, however, stereochemistry of the compound was revealed in 1994 when Ichihara and coworkers extensively worked on this class of compounds ${ }^{6-8}$. Due to its significant biological activity and intriguing molecular structure, efforts have been made to triumph its synthesis.

1.1 Synthesis by Ichihara and Co-workers:

Ichihara and co-workers were the first to report the synthesis of Alternaric acid $^{9}$. The synthesis of the targeted molecule was challenging in the sense that the stereochemistry of the desired natural product $\mathbf{1}$ at $\mathrm{C} 10$ and $\mathrm{C} 11$ was not previously established. In order to design the strategy for the synthesis of alternaric acid 1, it was required to get explicit evidence for the absolute configuration of the alternaric acid at above mentioned stereocenters. Thus, all the four diastereomers of C9-C14 fragment $\mathbf{2}$ of alternaric acid $\mathbf{1}$ were synthesized. To get confirmation of stereochemistry, output was equated with the degradation product of the natural sample. The stereochemistry of target product was successfully answered and was found to be syn diol. For the total synthesis of $\mathbf{1}$, Ichihara strategy involved the coupling of three components A, $\mathrm{B}$ and $\mathrm{C}$ as shown in the figure $\mathbf{2}$.<smiles>CC[C@H](C)C(OC(C)(C)C)C(C=O)OC(C)(C)C</smiles>

Component A

2<smiles>C=C(CCC=C[C@@](OC)(OC(C)(C)C)[C@H](OC(C)(C)C)[C@@H](C)CC)CCC[OH+]</smiles>

Component $\mathrm{AB}$

4

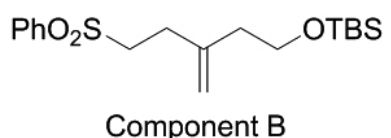

3<smiles>C[C@H]1CC(=O)CC(=O)O1</smiles>

Component C
Figure 2: A, B and C fragments of Alternaric Acid

Compound 2 (Component A) was synthesized by using commercially available starting material over ten steps. Their methodology involved the conversion of $(S)-(-)-2$-methylbutanol to $(S)-(+)-2$-methylbutanal 6 using standard conditions of Swern oxidation ${ }^{10}$ and resulting aldehyde 6 was treated with 7 to harvest the diasteromers $\mathbf{8 a}$ and $\mathbf{8 b}$ with a selectivity of 16:9. The resulting diaseteromeric mixture $\mathbf{8 a} \& \mathbf{8 b}$ was dihydroxylated with osmium tetraoxide using NMO. The stereochemical outcome was later confirmed by NOE experiments as well as by comparing it with the L-isoleucine. The mixture of $\mathbf{8 a} \& \mathbf{8 b}$ was further treated with TBDPSCl to selectively protect primary alcohol. This event set the stage for the formation of acetonide 10 \& 11 which was obtained in $72 \%$ yield from $\mathbf{6}$ in a mixture of four diastereomers with a selectivity of 60:34:6. Compounds $\mathbf{1 0}$ and $\mathbf{1 1}$ were isolated purified by flash chromatography. The stereochemistry of the resulting compound was revealed via NOE experiments and desired component 11 was identified.

In the next step, acetonide $\mathbf{1 1}$ was hydrolysed using silicon dioxide and trimethylsilyl chloride to generate aldehyde $\mathbf{1 2}$ which was further oxidized with sodium chlorite and then methylation with diazomethane. The silyl deprotection of the resulting methyl ester was performed by tetra butyl ammounium fluoride in THF and acetic acid. Acetic acid was employed to maintain the acidic environment in the presence of TBAF thus inhibited the hydrolysis of the methyl ester. Finally, the liberated alcohol $\mathbf{1 3}$ was subjected to Swern oxidation to accomplish the synthesis of component A 2. 


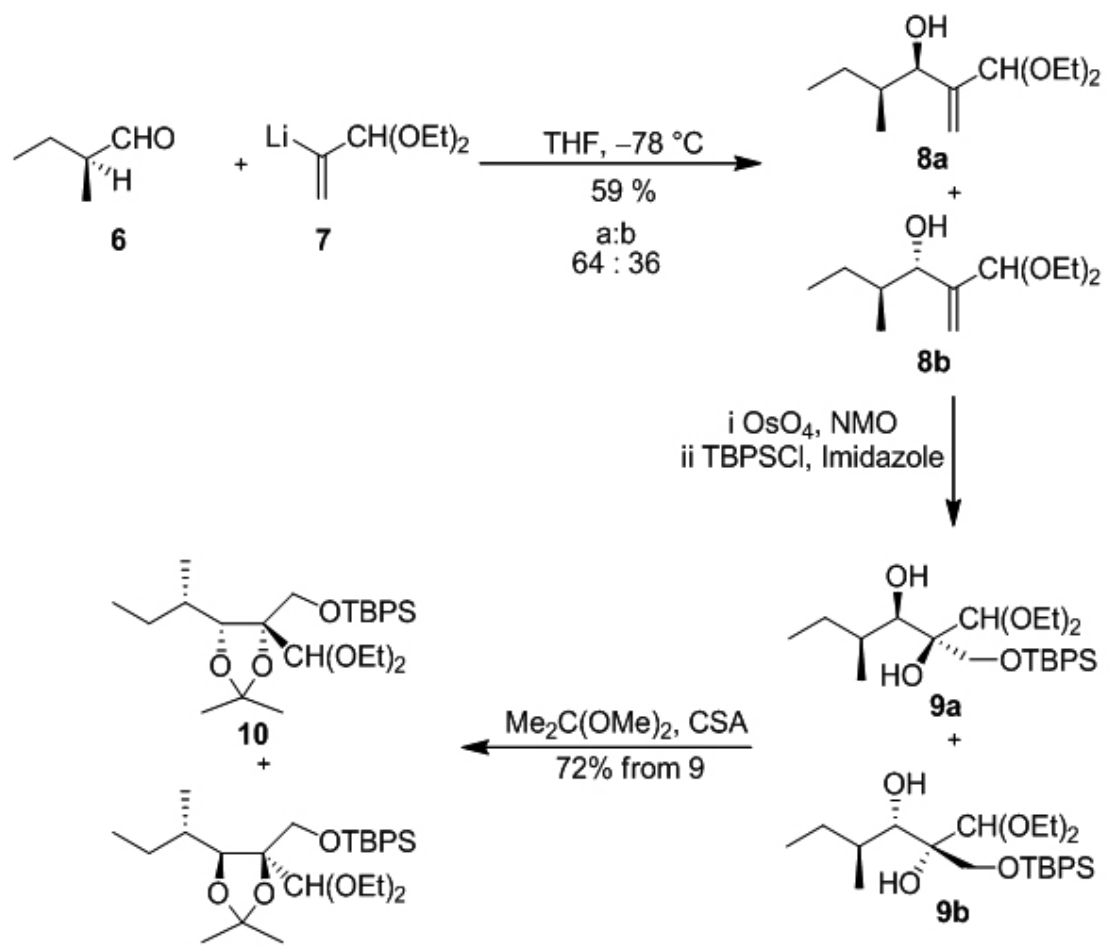

Scheme 1

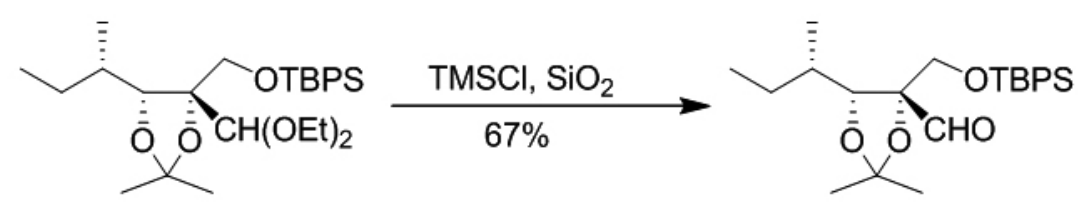

11

12

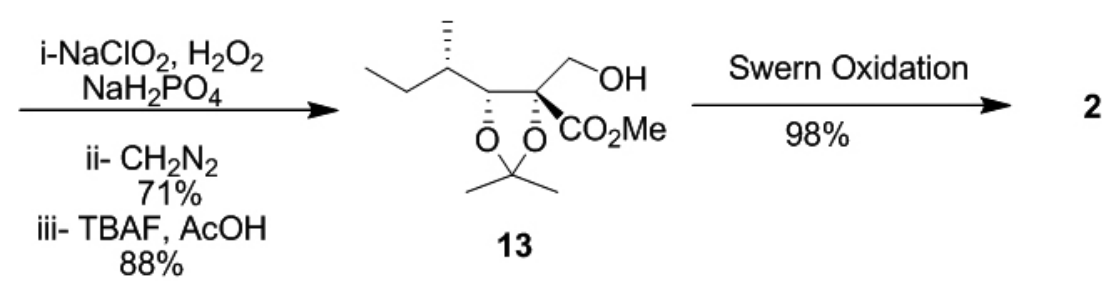

Scheme 2

In order to accomplish the synthesis of component B, dimethyl itaconate 14 was attempted to reduce toafford diol, however direct reduction did not work. Therefore, the situation was triumphed by the use of the retro-Diels Alder methodology in which olefin was protected using cylopentadiene which resulted in the formation of $\mathbf{1 6}^{11}$. It was followed by reduction with $\mathrm{LiAH}_{4}$ and resulting product 17 was subjected to acetylation and then subsequent heating gave rise to diacetate 18. In the next step, dimethyl ester $\mathbf{1 9}$ was synthesized using sodium dimethyl malonate by adopting Malleron procedure ${ }^{12}$. Ester 19 was further heated in DMSO with sodium chloride to furnish $\mathbf{2 0}^{13}$. To introduce phenylsulphonyl in 20, simple and high yielding functional group inter conversion was adopted to achieve the synthesis of component B 3. Compound 20 was converted to alcohol using $p$-toluene sulfonic acid in methanol. The resulting alcohol was then subjected to diphenyl disulfide and tri- $n$ - butylphosphine ${ }^{14}$. The outcome was further exposed with diphenyldiselenide and hydrogen peroxide to carry out selective oxidation of sulfide group to furnish phenylsulphon ${ }^{15}$ which was further reduced with lithuim aluminium hydride followed by protection with silyl group to provide component B $\mathbf{3}$ in $16 \%$ yield over 11 steps.

Component $\mathrm{C}$, for the synthesis of Alternaric Acid, was efficiently prepared over two steps by adopting literature procedure ${ }^{16}$. The first step involved the Claisen condensation of $(R)$-()-methyl-3-hydroxybutanoate 22 and lithium tertbutyl acetate $\mathbf{2 3}$ to furnish $\beta$-keto ester $\mathbf{2 4}$ in good yield. It was followed by stirring with trifluoric acid for $24 \mathrm{~h}$ to hydrolyze ketoester to offer component C 5 with overall $70 \%$ yield in just two steps. 


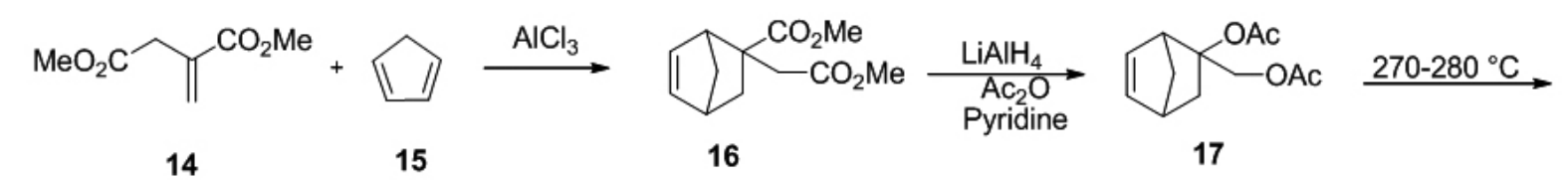

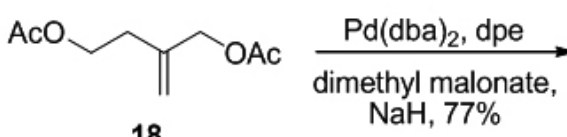

18

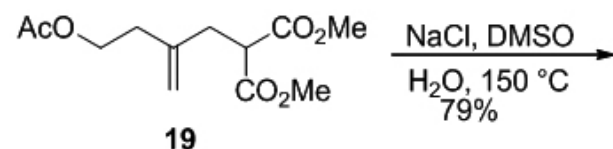

19<smiles>C=C(CCC(C)=O)CCC(C)=O</smiles>

20

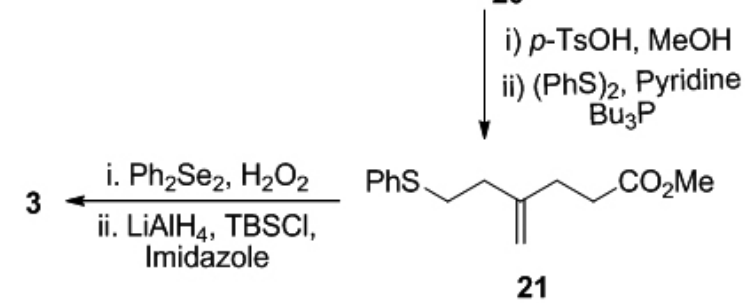

Scheme 3

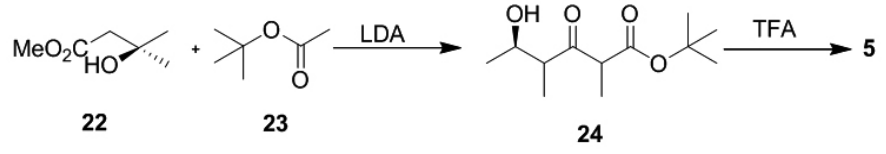

Scheme 4

In order to move towards the target molecule, component A $\mathbf{2}$ and B $\mathbf{3}$ was coupled using Julia olefination ${ }^{17}$. In this regard model experiment was devised to get clues about the conditions and outcome. It appeared that base and solvent both play important role in achieving good yield and LDA/ether- $n$-hexane $(1: 1)$ were found to give best results.

Therefore, coupling was performed using LDA in the solvent of $n$-hexane and ether in the ratio of 1:1 to offer $\beta$-hydroxyl suphone followed by acylation to make stage ready for elimination. It was performed by means of sodium amalgam to gain mixture of $E$ and $Z$ alkene 26 in a ratio of 14:1. The desired isomer 26 was isolated in considerable yield of $41 \%$ using MPLC technique. The cleavage of the silyl group of $\mathbf{2 6}$ was carried out by TBAF and subsequently exposed to Swern oxidation to yield aldehyde $\mathbf{2 8}$ which underwent further oxidation by sodium chlorite to yield carboxylic acid $\mathbf{2 9}$.

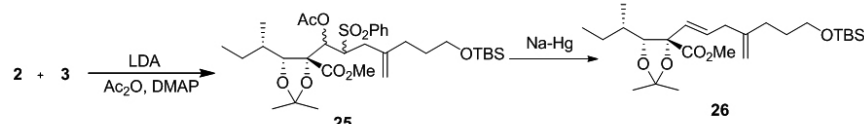

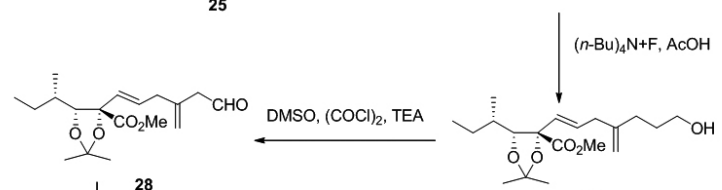

$$
\begin{aligned}
& \underbrace{\mathrm{COOH}}
\end{aligned}
$$

Scheme 5

At this stage, synthesis of carboxylic acid $\mathbf{2 9}$ and $o$-enolacylation of $\beta$-keto$\delta$-valerolactone (component C) $\mathbf{5}$ set a platform for Fries type rearrangement. To move towards the target molecule, the synthesis of the lactone $\mathbf{3 2}$ was desired. For the synthesis of desired molecule, several methods were available in the literature ${ }^{18}$, however, Ichihara and coworkers accomplished it by a novel one-pot construction using Fries type rearrangement from carboxylic acid and lactone to yield 3-acyl-4-hydroxy-5,6-dihydro-2-pyrone ${ }^{19}$. This reaction was as performed using DCC and DMAP to afford 3-acyl-4-hydroxy-5,6-dihydro-2- pyrone. In the event, hydrolysis of $\mathbf{3 2}$ did not prove much fruitful by the aid of $\mathrm{HCl}$ in methanol and THF and resulted in only $21 \%$ yield of desired compound and further hydrolysis could not result in, the target compound 1 . Thus, Icharia and co-workers accomplish it by using $2 \mathrm{~N}$ lithium hydroxide in a mixture of methanol-THF to hydrolyse methyl ester. The compound was further heated at $120{ }^{\circ} \mathrm{C}$ for the hydrolysis of acetonoide $\mathbf{3 3}$ to furnish final target $\mathbf{1}$ in overall $0.001 \%$ yield over 29 steps.

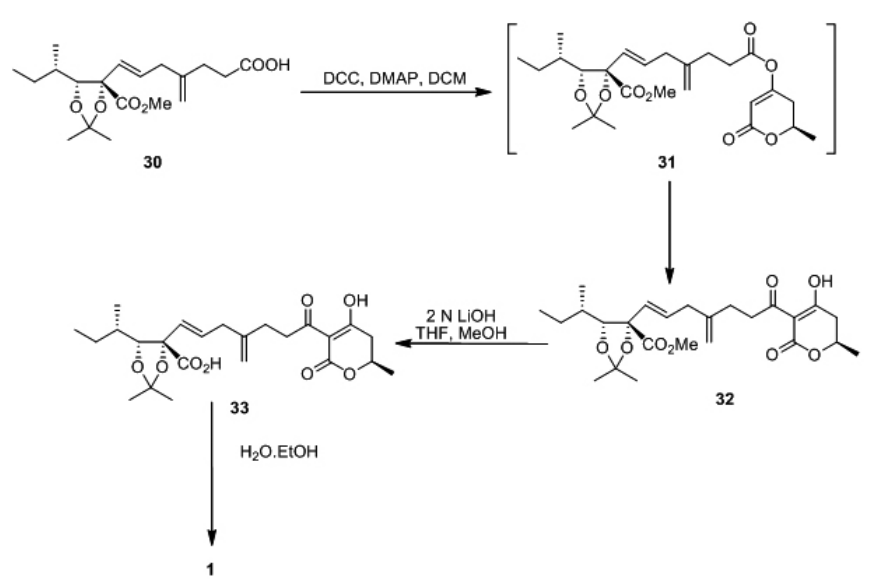

Scheme 6

1.2 Synthesis by B. M. Trost and co-workers:

In the synthesis of Alternaric acid, Trost utilized the Ru catalyzed Alder ene type reaction which proved very efficient and attractive approach in comparison to previous known synthesis ${ }^{20}$. Trost group devised two comparatively similar pathways (as shown in scheme 7) to exploit the role of Ru catalyzed coupling of terminal methylene and an (E)-1,2-disubstituted olefin. Both approaches made use of alkene 36, alkyne $\mathbf{3 7}$ and pyrones $\mathbf{3 5}$, thus their synthesis became comparable to that of Ichicharia. However, Trost methodology relied on two factors; a) the success application of their established Alder-ene methodology during coupling of ene and alkyne b) and the efficacy of their synthesis to proposed alkene 34

The model study was carried out via two pathways as described in scheme $7^{21}$. Their study involved the reaction of allylated derivatives with tert-butyl 4-pentynoate 37 using $5 \mathrm{~mol} \%$ of the ruthenium complex $\mathbf{4 0}$. The results revealed that the use of carboxylic acid substrate and acyldihydropyrones gave low yield (scheme 8). This appeared from the fact that both could cause good coordination anions that impeded Ruthenium activity during the process. The 
study elucidated that ruthenium catalyst exhibited limited tolerance for the carboxylic acid substrate in comparison to methyl ester and hydroxyl group substrates. In light of the above results, Trost decided to use path B for the synthesis alternaric acid $\mathbf{1}$ of scheme 7 which do not carry acyldihyropyrones during $\mathrm{Ru}$ catalyzed step.

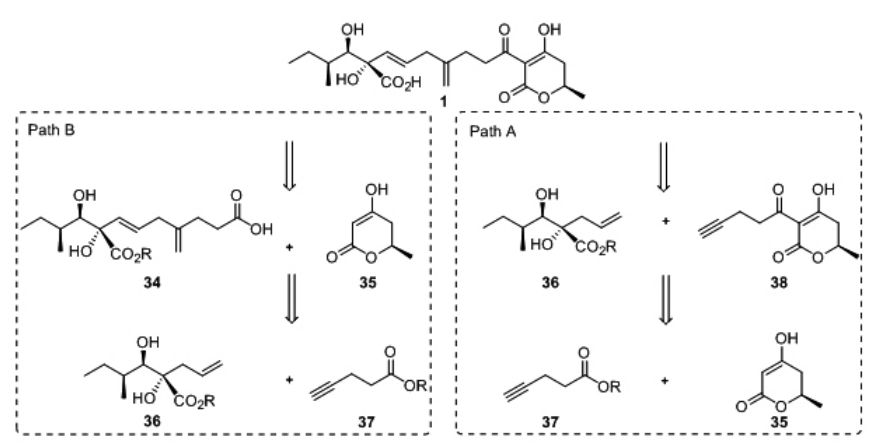

Scheme 7

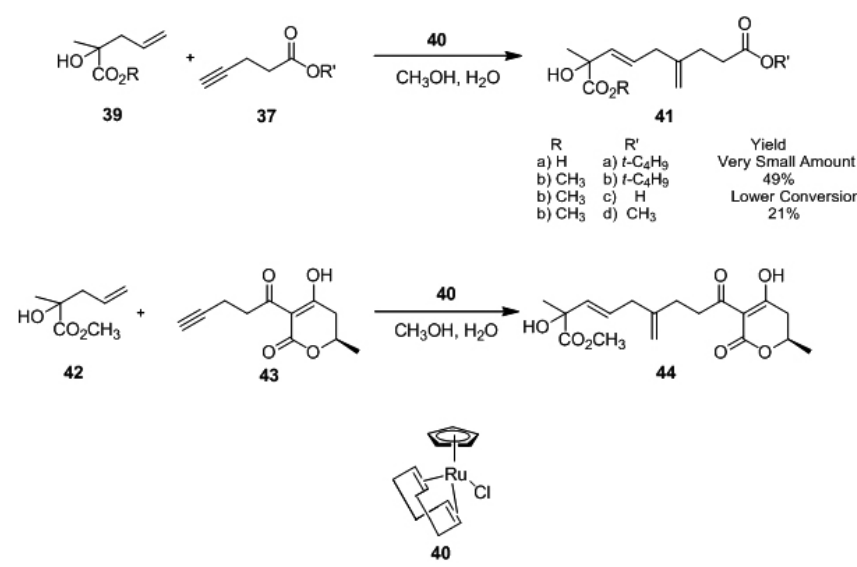

Scheme 8

In this path, efforts were made to construct alkene 34. They started with the commercially available enantiomerically pure alcohol which was subjected to oxidation to yield $\mathbf{4 5}$ followed by reaction with ylide $\mathbf{4 6}$ to hope for single geometric isomer of $E$-alkene $\mathbf{4 7} \mathbf{7}^{22}$. However, it yielded a racemized product $\mathbf{4 7}$ with diastereomeric ratio of $1.2: 1$. In order to improve the ratio, reaction was performed at $78^{\circ} \mathrm{C}$ to room temperature with different phosphonates like 48 a, $48 \mathrm{~b}$ and $48 \mathrm{c}$. The reaction furnished $E$ and $Z$ alkenes but ratio and yields of the desired isomer could not be improved.

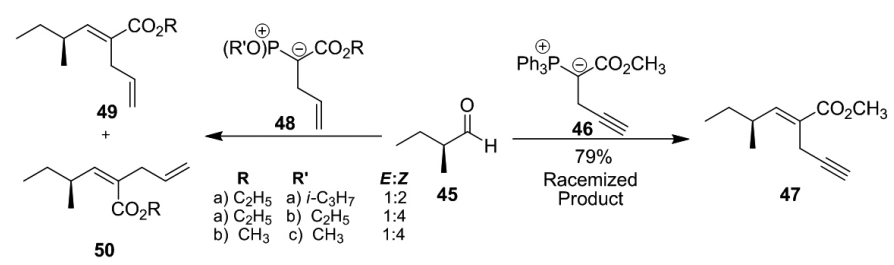

Scheme 9

In order to achieve better results, an alternative route proved successful in the synthesis of desired alkene. In this approach, alcohol $\mathbf{4 6}$ was oxidized using Moffat-Swern ${ }^{10}$ method followed by treatment with Wittig reagent to provide the alkene 47. The stereochemistry of resulting alkene was confirmed by NMR and was further subjected to bromination followed by dehydrobromination which led to the formation of single geometric isomer of vinyl bromide 48. Here, Trost and coworkers made use of Stille cross-coupling to enable the synthesis of $\mathbf{4 9}$ as a single geometric isomer ${ }^{23}$. In order to achieve the dihydroxylation, all the resulting substrates were subjected to osmium tetraoxide, however, none of these yielded in desired diol. Enyne 47 proved non reactive and did not undergo any reaction which was contrary to literature reports which showed trisubstituted alkenes having carbonyl could easily be dihydroxylated, whereas, $49 \mathrm{~b}$ provided dihydroxylated product but at its nonconjugated alkene ${ }^{24-25}$.

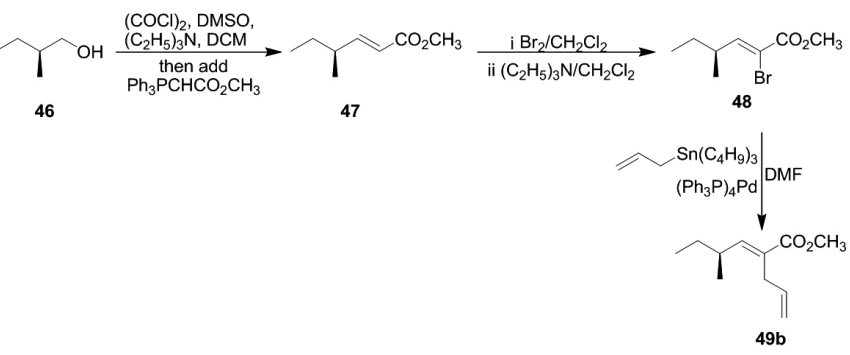

Scheme 10

In order to come out of this impediment, tert-butyldimethylsilyl ether of allyl alcohol was used as an alternative approach for the generation of substituted alkene. Thus, protected alcohol $\mathbf{5 0}$ was coupled with vinyl bromide 48 using one pot Suzuki cross coupling reaction to achieve a single isomer of $\mathbf{5 1}$ in $88 \%$ isolated yield ${ }^{26}$. At this stage, asymmetric dihydroxylation was smoothly carried out to obtain hydroxylated product $\mathbf{5 2}$ in $89 \%$ yield and $98 \%$ diastereomeric access. It was accompanied by acetonoid protection followed by desilylation and was subsequently subjected to Grieco method to provide desired alkene $\mathbf{3 6}^{27}$. The resulting alkene could also be achieved when triol was subjected to Grieco procedure which proceeded smoothly without compromising the yield, thus, saving one extra step and showing that acetonoid protection is not necessary at this stage. On the whole, desired alkene $\mathbf{3 6}$ was ultimately achieved in just seven steps from commercially available alcohol $\mathbf{4 6}$.

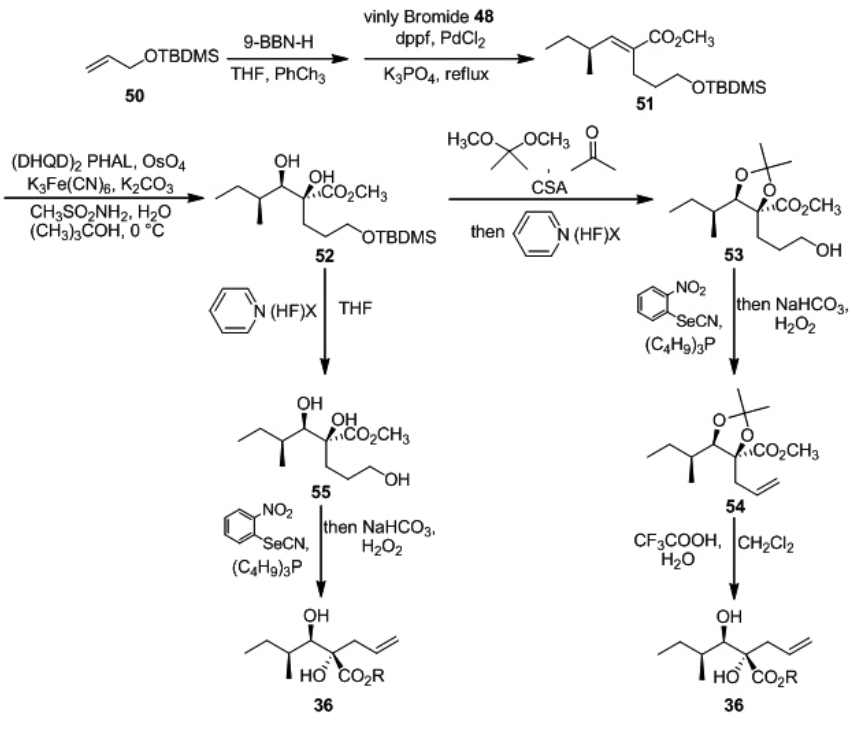

Scheme 11

In the next phase, alkyne moiety was easily synthesized which involved the alkylation of the enolate of tert-butyl acetate in THF with the help of LDA and HMPA along with propargyl bromide $\mathbf{5 6}$ to furnish tert-butyl ester in $66 \%$ yield. Here, tert-butyl ester of the alkyne moiety was further solvolysed with TFA in DCM to obtain acid $\mathbf{3 7 b}$. The resulting acid was esterified with diazomethane to generate desired methyl ester $\mathbf{3 7}$.

With two major constituents in hand, efforts were made to accomplish alder ene reaction of alkene and alkyne of tert-butyl ester. In this regard, mixture of alkene $\mathbf{5 4}$ and alkyne of tert-butyl ester 37a in 1:1 ratios was heated at $100{ }^{\circ} \mathrm{C}$ in the solvent mixture of DMF and water. This provided $26 \%$ yield of ene adduct $\mathbf{5 8}$ and $\mathbf{5 9}$ in ratio of 1.6 to 1 . To improve the yield and selectivity of branched to liner adduct, various additives were also used to aid the reaction, however, best result could yield $58 \%$ of the product in attenuated regioselectivity. 


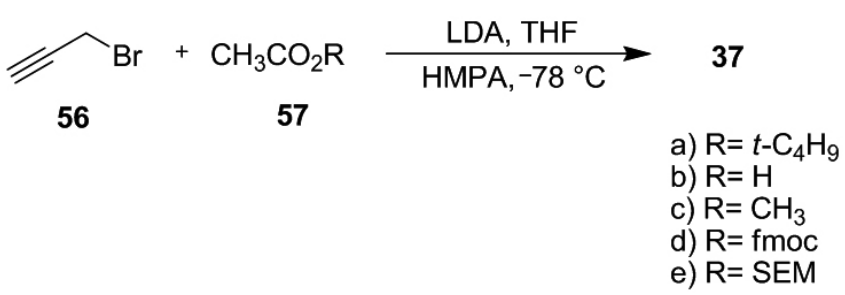

Scheme 12

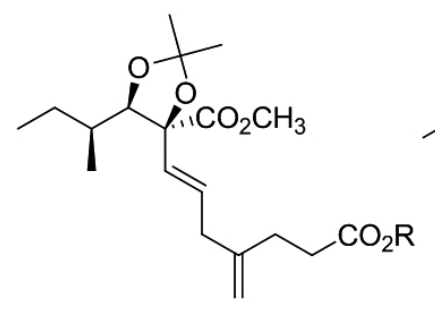

58

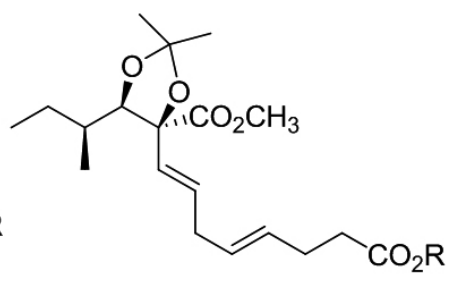

59
Figure 3: Structures of ene adduct $\mathbf{5 8}$ and $\mathbf{5 9}$

To improvise the desired results, coupling reaction was repeated using alkene 36 in pure methanol as a solvent. Several experiments were designed to optimize the reaction output by varying the catalyst load, additives type, ambient pressure, reaction time and temperature. These efforts resulted in comparatively better yield and selectivity for the desired ene adduct. At this stage, Trost and coworkers required $\mathbf{5 8 b}$ to complete the formal synthesis which required acetonide protection of diol fragment of $\mathbf{6 0}$. Therefore, reaction was carried out for the acetonide formation, however, it could not be hydrolyzed to yield the desired product as either it decomposed or showed chemoselectivity issues.<smiles>[R]OC(=O)CCC(=C)C/C=C/[C@@](O)(C(=O)OC)[C@@H](O)[C@@H](C)CC</smiles>

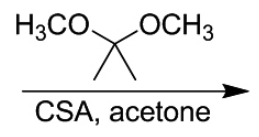

60

\section{8}

a) $\mathrm{R}=t-\mathrm{C}_{4} \mathrm{H}_{9}(90 \%)$ b) $\mathrm{R}=\mathrm{H}(0 \%)$ c) $\mathrm{R}=\mathrm{CH}_{3}(96 \%)$ d) $R=\operatorname{fmoc}(97 \%)$
Scheme 13

To come out of this hurdle and to get the end point of this formal synthesis, Trost and coworkers selected SEM and fmoc esters to mask the acid. Thus, both SEM and fmoc esters were coupled with 4-pentynoic acid in the presence of DCC and DMAP to yield respective esters in $85 \%$ and $71 \%$ yield (scheme 12). At this point, both esters were subjected to ruthenium catalyzed coupling conditions to get SEM or Fmoc protected ene adduct. Studies showed that SEM protected substrate did not furnish any desired product, however, Fmoc ester showed satisfactory results. Excessive efforts were carried out to optimize the conditions that provided excellent yield and maximized the output of the branched product $\mathbf{6 0}$ verses linearly-linked product $\mathbf{6 1}$.

They could easily cleave fmoc ester $\mathbf{6 0 d}$ by using piperidine in ethylene chloride (scheme 14) which furnished desired $\mathbf{5 8 b}$ in admirable yield which could, then, be coupled with dihydropyrone $\mathbf{5}$ followed by hydrolysis to triumph the alternaric acid synthesis.

The utilization of Trost-Ru-catlysed coupling of ene and terminal alkyne provided a powerful strategy which allowed completing the synthesis of ene fragment over 11 steps with overall yield of $27 \%$ in comparison to that of Ichicharia approach which furnished the same in 26 steps and $0.003 \%$ yield.

1.3 Synthesis by Slade and Johnson:

Slade and Johnson exploited the role of silylglyoxylate for variety of synthetic activities which involved successful introduction of electrophilic and nucleophilic subunits on glycolic moiety to build a molecular complexity ${ }^{28}$.
They also employed their silylglyoxylates for the formal synthesis of Alternaric acid in which functional group and desired stereochemistry was installed on glycolic acid to attain basic core of desired target (scheme 15).

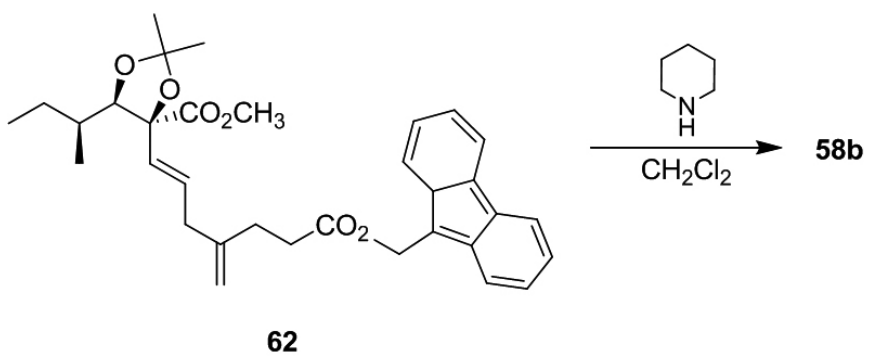

Scheme 14

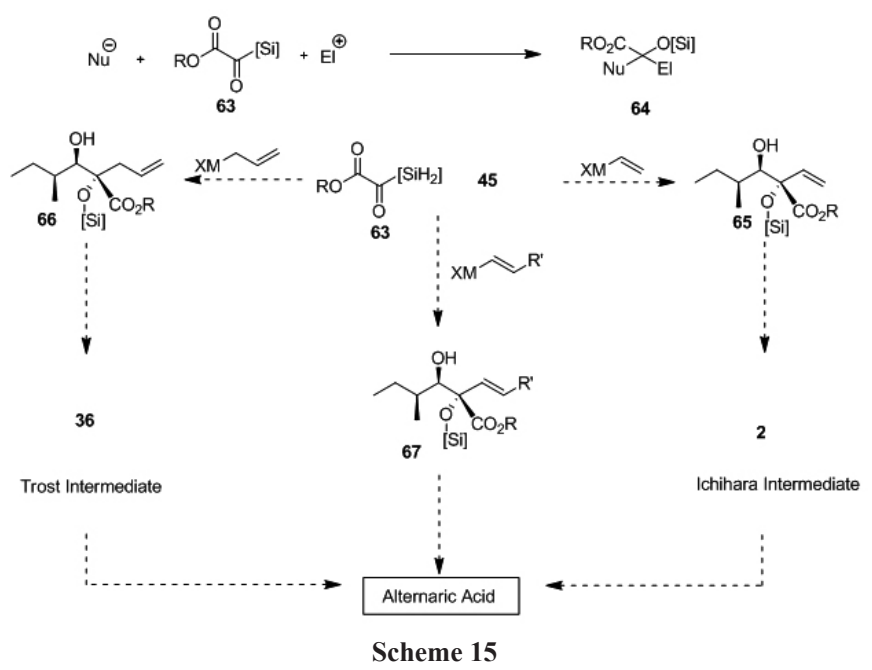

They devised two analogous strategies for the synthesis of Alternaric Acid; coupling (S)-2-methylbutanal $\mathbf{4 5}^{22}$ in one case and silylglyoxylate $\mathbf{6 3}$ in the other with vinyl and allyl Grignard ${ }^{28}$. In their first approach, reactions were proceeded in the presence of (-)-sparteine using vinyl Grignard 69 to yield coupling product 70 in $65 \%$ yield and $>20: 1$ syn/anti aldol and 1.7:1 facial selectivity. In order to obtain Icharia aldehyde, intermediate product 70 was warmed in acidic methanol to effect silyl cleavage and methyl ester formation. Aldol was further protected using acetonide formation which was followed by ozonolysis to furnish Ichihara's aldehyde 2 . To get the olefin from aldehyde, phenyl tetrazole on sulphone $\mathbf{7 3}$ was employed during modified Julia olefination to achieve the synthesis of $\mathbf{7 4}^{29}$.

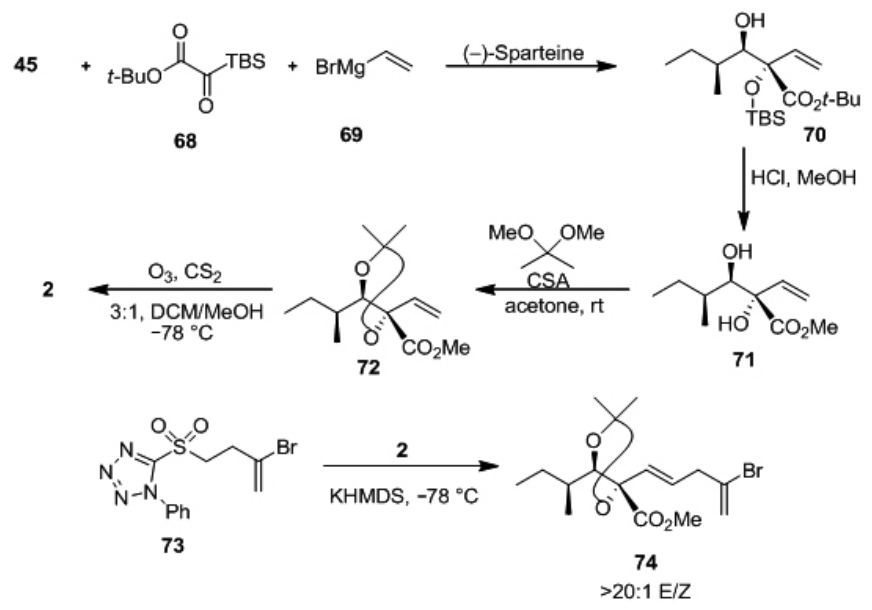

Scheme 16 
In their complementary approach to alternaric acid, allyl Grignard $\mathbf{7 5}$ was tried along with silylglyoxylate $\mathbf{6 8 b}$ and $(S)$-2-methylbutanal $\mathbf{4 5}$ to gain coupling adduct 76. In this process $>3.6: 1$ syn/anti selectivity and $1.7: 1$ facial selectivity was achieved, however, all the four diastereomers could not be separated.

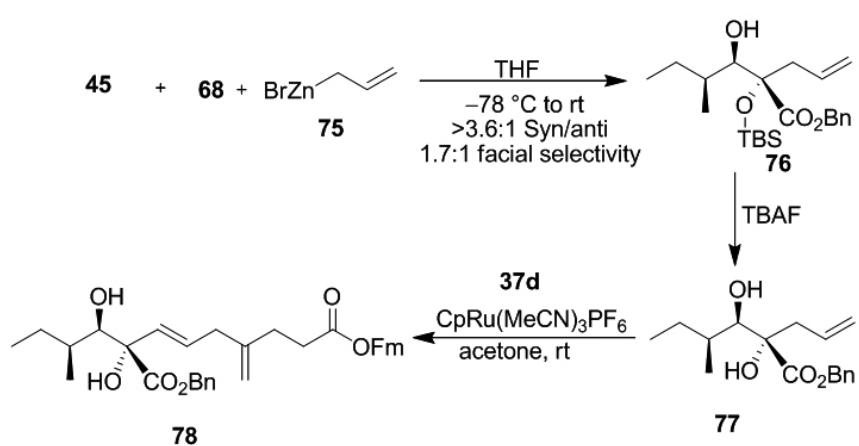

Scheme 17

The coupling product $\mathbf{7 6}$ was deprotected with TBAF to yield benzyl ester analogue of the Trost substrate. This aldol was successfully coupled with Fmoc ester of alkyne $37 \mathbf{d}$ by employing the $\mathrm{CpRu}(\mathrm{MeCN})_{3} \mathrm{PF}_{6}$ in $52 \%$ yield.

In both above mentioned strategies, they successfully exploited the role of (S)-2-methylbutanal and silylglyoxylate in the three component coupling adduct which were subjected to functional group manipulations to yield basic core of desired end point but these efforts could not result in better facial selectivity. However, all the endeavors for the separation of diastereomers could meet with no sign of sought. Upon realization, Johnson and coworkers designed several other approaches to accost the stereochemical outcomes. All of these involve the introduction of auxiliary modification of silylyglyoxylate and aldehyde moiety as shown in figure $3^{30}$.

Modified Silyl glyoxylate<smiles>[R]CC1CCCC(O[Z20])C1c1ccccc1</smiles>

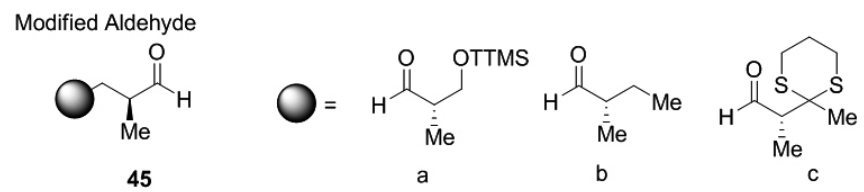

Figure 3

However, three component coupling adduct was formed in all cases, but, yield and stereochemical output could not be achieved to the desired level unless 1,3-dithiane group in aldehyde $\mathbf{4 5} \mathbf{c}$ was introduced during the racemic synthesis of three component coupling product. It resulted in high yield along with excellent stereochemical outcome $>20: 1^{31-32}$. In the subsequent step, dithiane $\mathbf{8 0}$ was cleaved followed by 1,3-syn selective reduction to yield diol $\mathbf{8 2}$ which under acidic conditions provided $\mathbf{8 3}^{33}$.

The use of the dithiane aldehyde $45 \mathrm{c}$ convinced Johnoson and coworkers for the formation of more functionalized vinyl nucleophile to assemble carbon back bone of the alternaric acid in a single three component coupling step. Thus, they designed another approach to keep the route towards desired alternaric acid further short as well as to avoid additional step for removal of directing groups. In this approach, they used more functionalized nucleophile to install major skeleton of the alternaric acid via three component coupling reaction scheme 19 .

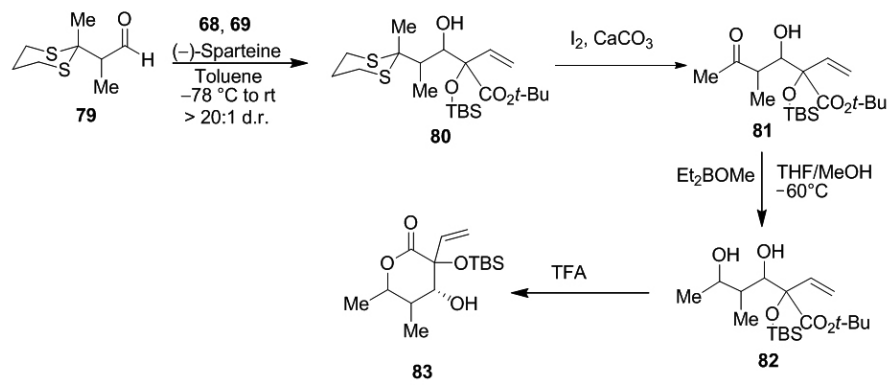

Scheme 18

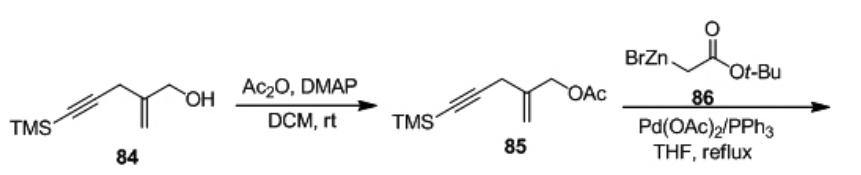

$\underset{87}{\stackrel{\mathrm{TBAF}, \mathrm{NH}_{4} \mathrm{Cl}}{\mathrm{OCM}, \mathrm{rt}, 95^{\circ} \mathrm{C}}}$

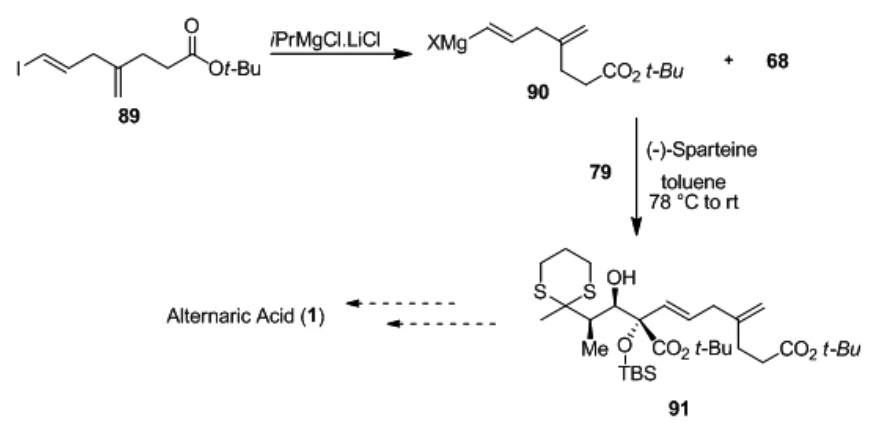

Scheme 19

Thus, allylic alcohol 84 was acylated to afford ester $\mathbf{8 5}$ followed by treatment with Reformatsky reagent to yield $\mathbf{8 7}^{34}$. Here, buffered TBAF was utilized to remove silyl group. It was, then, subjected to hydrozirconation/ iodination by employing Schwartz's reagent ${ }^{35}$ to generate vinyl iodide 89 . In order to achieve the synthesis of vinyl nucleophile 90, Knochel's $\mathrm{Mg} / \mathrm{I}$ exchange reaction was carried out $^{36}$. Here, one pot tandem reaction was performed by employing vinyl nucleophile $\mathbf{9 0}$, silylglyoxylate $\mathbf{6 8}$ and dithiane aldehyde $\mathbf{4 5 c}$ for the highly convergent three component coupling product 91. To complete the $3^{\text {rd }}$ approach for the formation of natural product $\mathbf{1}$, functional group manipulation like desulfurization and deprotection of silyl and tert-buty ester groups are required which was followed by addition of pyrone moiety 5 as described by Ichicharia'. Thus, in this way, Slade and Johnson successfully exploited the role of silylglyoxylate and functionalized electrophile and nucleophile for the development of three short and diverse routes to the total synthesis of alternaric acid.

\subsection{CONCLUSION:}

In conclusion, variety of strategies has been devised by Ichihara, Trost and Johnson groups towards the total and formal synthesis of Alternaric Acid. All of them synthesized different fragments of target molecule and then coupled it together for the desired outcome. Ichihara approach involved modified Julia olefination for the synthesis of acyclic fragment of alternaric acid over 26 steps in $0.003 \%$ whereas Trost strategy constructed the same fragment via Ruthenium catalyzed coupling step over 11 steps and $27 \%$ yield, however, both started from (S)-2-methyl-1-butanol. In comparison, Johnson made use of silylgylyoxylates along with different electrophilic and nucleophilic moieties to furnish desired targets in relatively clean and short way.

In order to complete the synthesis of desired targets, synthetic practitioners should always be mindful of the flexibility and creativity for the precise organic transformations. Such inspiration in choosing the approach could lead to even 
more striking novelty and spectacular elegance.

\subsection{ACKNOWLEDGEMENT:}

The authors are thankful to Government College University Faisalabad, University of Engineering and Technology, Lahore and Higher Education Commission, Pakistan for providing facilities to carry out this work.

\section{REFERENCES:}

1. P. W. Brian, P. J. Curtis, H. G. Hemming, C. H. Unwin and J. M. Wright, Nature, 164, 534 (1949)

2. P. W. Brian, P. J. Curtis, H. G. Hemming, E. G. Jefferys, C. H. Unwin and J. M. Wright, J. Gen. Microbiol., 5, 619 (1951)

3. P. W. Brian, G. W. Elson, H. G. Hemming and J. M. Wright, Ann. Appl. Biol., 39, 308 (1952)

4. J. F. Grove, J. Chem. Soc. , 4056 (1952)

5. N. Furuichi, S. Nishimura and G. Langsdorf, Ann. Phytopathol. Soc. Jpn., 58, 1 (1992)

6. J. R. Bartels-Keith, J. Chem. Soc., 860 (1960)

7. J. R. Bartels-Keith, J. Chem. Soc., 1662 (1960)

8. H. Tabuchi, H. Oikawa and A. Ichihara, J. Chem. Soc. Perkin Trans.1, 2833 (1994)

9. H. Tabuchi, T. Hamamoto, S. Miki, T. Tejima and A. Ichihara, J. Org. Chem., 59, 4749 (1994)

10. A. J. Mancuso, S.-L. Huang and D. Swern, J. Org. Chem., 43, 2480 (1978)

11. A. Ichihara, Synthesis, 1987, 207 (1987)

12. J. C. Fiaud and J. L. Malleron, Tetrahedron Lett., 21, 4437 (1980)

13. A. G. Brook, Acc. Chem. Res., 7, 77 (1974)

14. I. Nakagawa and T. Hata, Tetrahedron Lett., 16, 1409 (1975)

15. M. P. Edwards, S. V. Ley, S. G. Lister, B. D. Palmer and D. J. Williams, J. Org. Chem., 49, 3503 (1984)
16. P.-F. Deschenaux, T. Kallimopoulos, H. Stoeckli-Evans and A. JacotGuillarmod, Helv. Chim. Acta, 72, 731 (1989)

17. M. Julia and J.-M. Paris, Tetrahedron Lett., 14, 4833 (1973)

18. Y. Tanabe, M. Miyakado, N. Ohno and H. Yoshioka, Chem. Lett., 11, $1543(1982)$

19. H. Tabuchi, T. Hamamoto and A. Ichihara, Synlett, 1993, 651 (1993)

20. T. Ohe, N. Miyaura and A. Suzuki, J. Org. Chem., 58, 2201 (1993)

21. G. D. P. Barry M. Trost, and Andreas Schoop, J. Am. Chem. Soc., 9228 (1998)

22. R. S. Atkinson and M. J. Grimshire, J. Chem. Soc. Perkin Transactions 1 , 1215 (1986)

23. P. L. Anelli, Montanari F., Quici S, Org. Synth., 69, 212 (1990)

24. J. K. Stille, Angew. Chem., Int. Ed. Engl., 25, 508 (1986)

25. H. C. Kolb, M. S. VanNieuwenhze and K. B. Sharpless, Chem. Rev., 94, $2483(1994)$

26. P. G. Andersson and K. B. Sharpless, J. Am. Chem. Soc., 115, 7047 (1993)

27. P. A. Grieco, S. Gilman and M. Nishizawa, J. Org. Chem., 41, 1485 (1976)

28. M. C. Slade and J. S. Johnson, Beilstein J. Org. Chem., 9, 166 (2013)

29. P. R. Blakemore, J. Chem. Soc. Perkin Transactions 1, 2563 (2002)

30. T. Gaich and P. S. Baran, J. Org. Chem., 75, 4657 (2010)

31. H. Kim, J. B. Baker, S.-U. Lee, Y. Park, K. L. Bolduc, H.-B. Park, M. G. Dickens, D.-S. Lee, Y. Kim, S. H. Kim and J. Hong, J. Am. Chem. Soc., 131, $3192(2009)$

32. D. E. Ward and A. Kazemeini, J. Org. Chem., 77, 10789 (2012)

33. K.-M. Chen, K. G. Gunderson, G. E. Hardtmann, K. Prasad, O. Repic and M. J. Shapiro, Chem. Lett., 16, 1923 (1987)

34. J.-L. Renaud, C. Aubert and M. Malacria, Tetrahedron Lett., 40, 5015 (1999)

35. H. A. Reichard, J. C. Rieger and G. C. Micalizio, Angew. Chem., Int. Ed., 47, 7837 (2008)

36. H. Ren, A. Krasovskiy and P. Knochel, Org. Lett., 6, 4215 (2004) 